\title{
A REMARK ON CHAIN-HOMOTOPY
}

TIBOR RADO

1. Introduction. Let $X, Y$ be finite abstract complexes in the sense of [1] (numbers in square brackets refer to the bibliography at the end of this note). Integral coefficients will be used throughout in dealing with the homology groups attached to complexes. Two chainmappings $\tau_{1}, \tau_{2}$ are termed chain-homotopic, in symbols $\tau_{1} \simeq \tau_{2}$, if there exists, for each dimension $p$, a homomorphism

$$
D: \quad C_{p}(X) \rightarrow C_{p+1}(Y)
$$

such that

$$
\partial D c_{p}^{X}+D \partial c_{p}^{X}=\tau_{2} c_{p}^{X}-\tau_{1} c_{p}^{X}
$$

for every $p$-chain $c_{p}^{X}$ of the complex $X$. If $\tau_{1} \simeq \tau_{2}$, then $\tau_{1} \sim \tau_{2}$ (that is, $\tau_{1}, \tau_{2}$ induce identical homomorphisms from the homology groups of $X$ into the homology groups of $Y$ ). It is well known that the converse is generally false. Several examples to this effect may be found in [2]. A very simple example is given in $\$ 2$ below. On the other hand, if $X$ is torsion-free, then the relation $\tau_{1} \sim \tau_{2}$ does imply the relation $\tau_{1} \simeq \tau_{2}$ (see [1, pp. 155-157]). The purpose of this note is to further clarify the role of torsion in this connection, by proving the following result concerning simplicial complexes.

THEOREM. If the finite simplicial complex $K$ is not torsion-free, then there exists a finite simplicial complex $L$ and a pair of chain-mappings $\tau_{1}, \tau_{2}: K \rightarrow L$, such that $\tau_{1}, \tau_{2}$ are homologous but not chain-homotopic.

2. Preliminary remarks on finite abstract complexes. Even though our theorem is concerned with the simplicial case, clarity will be gained by developing part of the argument for finite abstract complexes. Let us denote by $C_{p}(X), Z_{p}(X), B_{p}(X), H_{p}(X)$ the groups of integral chains, cycles, boundaries, and the homology groups of the finite abstract complex $X$ (see [1]). The following points should be remembered in working with these concepts.

(a) If a certain dimension $p$ is missing, then the group $C_{p}(X)$ is defined as consisting of a zero-element alone. As a consequence, every $(p+1)$-chain is then a cycle, and the only $(p-1)$-boundary is the 0-element of $C_{p-1}(X)$.

(b) The incidence-numbers $\left[x_{p}: x_{p-1}\right]$ are not required to have only

Received by the editors May 8, 1950. 
the values $0,+1,-1$, although this happens to be the case in many important special instances.

Now let us consider the following example (abstracted from the case of the projective plane). Let $X$ consist of a single 1-cell $x_{1}$ and a single 0 -cell $x_{0}$, with the incidence-number $\left[x_{1}: x_{0}\right]=2$. Let $Y$ consist of a single 1-cell $y_{1}$ and a single 0 -cell $y_{0}$, with the incidencenumber $\left[y_{1}: y_{0}\right]=0$. The following statements are readily verified.

(i) $X$ and $Y$ are abstract complexes in the sense of [1], with the boundary relations $\partial x_{1}=2 x_{0}, \partial y_{1}=0$.

(ii) On defining $\tau: X \rightarrow Y, \sigma: X \rightarrow Y$ by the relations

$$
\tau x_{1}=y_{1}, \quad \tau x_{0}=0, \quad \sigma x_{1}=0, \quad \sigma x_{0}=0,
$$

both $\tau$ and $\sigma$ are chain-mappings, and $\tau \sim \sigma$.

(iii) On the other hand, the relation $\tau \simeq \sigma$ does not hold. Indeed, assume that $\tau \simeq \sigma$. Then there would exist a homotopy operator $D: C_{p}(X) \rightarrow C_{p+1}(Y)$ satisfying the relation $\partial D c_{p}^{X}+D \partial_{p}^{X}-\tau c_{p}^{X}=0$. In particular, we would have

$$
\partial D x_{1}+D \partial x_{1}-\tau x_{1}=0 .
$$

Now $D x_{1}=0$, since $Y$ has no 2-cells, and $D \partial x_{1}=2 D x_{0}$. Here $D x_{0}$ is a 1 -cell of $Y$, hence $D x^{0}=k y_{1}$, where $k$ is an integer. There follows the relation $(2 k-1) y_{1}=0$, a contradiction, since the integer $2 k-1$ is odd and hence different from zero.

This example shows that homologous chain-mappings need not be homotopic (as stated in the introduction, examples to this effect were constructed already in [2]). In constructing the above example, we took advantage of the fact that the incidence-numbers in a finite abstract complex are not restricted to the values $0,+1,-1$. However, as the theorem stated in the introduction shows, the same phenomenon arises in the case of simplicial complexes where the incidence-numbers are restricted to the values $0,+1,-1$.

3. A special chain-mapping for finite abstract complexes. Let $X$ be a finite abstract complex. We shall set up presently a special chain-mapping $\tau: X \rightarrow X$. For clarity, we shall use $\tau_{p}$ to refer to $\tau$ acting in dimension $p$. If a certain dimension $p$ is missing, then according to the conventions stated above the group $C_{p}(X)$ consists of a zero-element alone, and then $\tau_{p}$ is the trivial homomorphism carrying zero into zero. Accordingly, $\tau_{p}$ is thought of as acting in all dimensions $-\infty<p<+\infty$. We assert that there exists a set of homomorphisms $\tau_{p}: C_{p}(X) \rightarrow C_{p}(X)$, such that the following conditions hold.

(i) $\partial \tau_{p} c_{p}=\tau_{p-1} \partial c_{p},-\infty<p<\infty$. 
(ii) If $c_{p} \in B_{p}(X)$, then $\tau_{p} c_{p}=c_{p},-\infty<p<\infty$.

(iii) If $X$ is torsion-free in a certain dimension $p$, then $\tau_{p} z_{p} \in B_{p}(X)$ for every cycle $z_{p}$ in that dimension.

(iv) If $X$ does have torsion in a certain dimension $p$, then $\tau_{p} c_{p}=c_{p}$ for every $p$-chain $c_{p}$ in that dimension.

In the preceding statements, $B_{p}(X)$ denotes the group of $p$-boundaries in $X$. The existence of $\tau_{p}$ will be proved by induction.

Part 1. Let $p$ be an integer such that $\tau_{q}$ can be defined in all dimensions $q<p$ as required. Then $\tau_{p}$ can also be defined as required, without changing the homomorphisms $\tau_{q}$ in the dimensions $q<p$. We divide the proof of this assertion into two sections.

Case I. $X$ does have torsion in dimension $p$. In view of condition (iv), we have then no choice but to set $\tau_{p} c_{p}=c_{p}$, for every $p$-chain $c_{p}$ of $X$. Then (iv) holds, (ii) is obvious, (iii) is vacuous, and (i) is verified as follows. We have now $\partial \tau_{p} c_{p}=\partial c_{p}$. On the other hand, since $\partial c_{p} \in B_{p-1}$, the induction assumption (ii) applies with $q=p-1$, yielding $\tau_{p-1} \partial c_{p}=\partial c_{p}$. Thus $\partial \tau_{p} c_{p}=\tau_{p-1} \partial c_{p}=\partial c_{p}$.

Case II. $X$ is torsion-free in dimension $p$. Taking first the general case when the groups $C_{p}(X), Z_{p}(X), B_{p}(X)$ do not reduce to the zeroelement alone, we choose a canonical base for $C_{p}(X)$ (see [1, pp. 102103]). As a matter of fact, for our present purposes it is sufficient to observe that since $X$ is torsion-free in the dimension $p$ under consideration, we can choose for $C_{p}(X)$ a base consisting of three aggregates $b_{p}^{i}, z_{p}^{j}, c_{p}^{k}$ of $p$-chains such that the $b_{p}^{i}$ form a base for $B_{p}(X)$, the $b_{p}^{i}$ and the $z_{p}^{j}$ form a base for $Z_{p}(X)$, and of course the $b_{p}^{i}, z_{p}^{j}, c_{p}^{k}$ form a base for $C_{p}(X)$. The existence of such a base is due of course to the fact that by assumption the $p$-dimensional torsion-coefficients all vanish. Now consider $\partial c_{p}^{k}$. This being a boundary in dimension $p-1$, by the induction assumption (ii), applied with $q=p-1<p$, we have

$$
\tau_{p-1} \partial c_{p}^{k}=\partial c_{p}^{k}
$$

We now define $\tau_{p}: C_{p}(X) \rightarrow C_{p}(X)$ by assigning the images under $\tau_{p}$ of $b_{p}^{i}, z_{p}^{j}, c_{p}^{k}$ as follows:

$$
\tau_{p} b_{p}^{i}=b_{p}^{i}, \quad \tau_{p} z_{p}^{j}=0, \quad \tau_{p} c_{p}^{k}=c_{p}^{k} .
$$

We have to check the conditions (i)-(iv). The conditions (ii), (iii) are obviously satisfied in view of (2), condition (iv) is now vacuous, and condition (i) is verified as follows. Clearly, we have to check only that (i) holds for each of the $b_{p}^{i}, z_{p}^{j}, c_{p}^{k}$. Now since $b_{p}^{i}$ is a boundary, we have $\partial b_{p}^{i}=0$ and hence $\tau_{p-1} \partial b_{p}^{i}=0$, and by (2) also $\partial \tau_{p} b_{p}^{i}=\partial b_{p}^{i}=0$. 
Similarly, $\partial z_{p}^{j}=0$, hence $\tau_{p-1} \partial z_{p}^{j}=0$, and by (2) also $\partial \tau_{p} z_{p}^{i}=\partial 0=0$. Finally, by (1) and (2), $\partial \tau_{p} c_{p}^{k}=\partial c_{p}^{k}=\tau_{p-1} \partial c_{p}^{k}$.

Thus Case II is disposed of in the general case. If the $b_{p}^{z}$ are missing, or if both the $b_{p}^{i}$ and the $z_{p}^{j}$ are missing, and so forth, the same argument applies with trivial modifications.

Part II. Since $X$ is finite, we can choose an integer $p_{0}$ such that all dimensions $q<p_{0}$ are missing. For $q<p_{0}, C_{q}(X)$ reduces then to a zero-element, and we define of course $\tau_{q}$ as the trivial homomorphism $\tau_{q} 0=0$. The conditions (i)-(iv) manifestly hold then for all $q<p_{0}$. By Part I, we can therefore extend the definition of $\tau$, subject to the conditions (i)-(iv), successively to the dimensions $p_{0}, p_{0}+1, \cdots$. As a matter of fact, since $X$ is finite, we arrive after a finite number of steps at a dimension $q$ such that $C_{p}(X)$ reduces to a zero-element for all $p>q$. For all $p>q$, the trivial homomorphism $\tau_{p} 0=0$ satisfies then the conditions (i)-(iv). Thus our induction process is actually finite.

4. Preliminary remarks on simplicial complexes. Let $K$ be a nonempty finite simplicial complex. We take an auxiliary vertex $v$ not occurring in $K$, and arrange the vertices of $K$ in an arbitrary order $v_{1}, \cdots, v_{m}$. We then agree to use in the sequel the arrangement

$$
v, v_{1}, \cdots, v_{m}
$$

for purposes of orienting simplexes. In particular, the groups $C_{p}(K)$, $Z_{p}(K), B_{p}(K), H_{p}(K)$ will be set up in terms of the particular ordering (3). Now let $S$ be a nonempty subcomplex of $K$, and let $\Gamma$ be the cone over $S$ with vertex $v$. The simplexes of $\Gamma$ are oriented in terms of the ordering (3). We consider next the simplicial complex $L=K \cup \Gamma$, and we denote by $\eta: K \rightarrow L$ the injection from $K$ into $L$ and by $\lambda: K$ $\rightarrow L$ the zero-homomorphism. That is, $\lambda$ carries every element of $C_{p}(K)$ into the zero-element of $C_{p}(L)$, for every $p$. All these simplicial complexes are taken unaugmented. Accordingly, all negative dimensions are missing, and every 0 -chain is a cycle. Furthermore, all these simplicial complexes are subject to the conventions stated above for abstract complexes. The following well known facts will be used.

(a) If a cycle $z_{p}^{k}$ of $K$ lies in $S$, then $z_{p}^{k}$ bounds in $\Gamma$, provided that $p \geqq 1$.

(b) If $\beta$ is the dimension of $S$, and if $c_{\beta+2}^{L}$ is a $(\beta+2)$-chain of $L$, then we have a $(\beta+2)$-chain $d_{\beta+2}^{k}$ of $K$ such that $\eta d_{\beta+2}^{k}=c_{\beta+2}^{L}$. Indeed, since the dimension of the cone $\Gamma$ over $S$ is $\beta+1$, all the simplexes of $c_{\beta+2}^{L}$ occur in $K$ (as a matter of fact, $d_{\beta+2}^{k}$ is merely $c_{\beta+2}^{L}$ considered as a chain in $K$ ). 
5. Proof of the theorem stated in the introduction. Let $K$ be a finite simplicial complex which is not torsion-free, and let $\beta$ be the highest dimension in which torsion occurs. Let $S$ consist of all the simplexes of $K$ with dimension less than or equal to $\beta$, and let $\Gamma$ be the cone over $S$ with vertex $v$, where the conventions stated in $\$ 4$ are used. Let $\tau: K \rightarrow K$ be the special chain-mapping described in $\S 3$. We denote, as in $\$ 4$, by $\eta$ the injection from $K$ into $L$, and by $\lambda$ the zero homomorphism from $K$ into $L$. We have then the chainmappings

$$
\sigma_{1}=\eta \tau: K \rightarrow L, \quad \sigma_{2}=\lambda \tau: K \rightarrow L .
$$

We assert that $\sigma_{1}$ and $\sigma_{2}$ are homologous but not chain-homotopic. Since $\sigma_{2}$ is the zero chain-mapping, we have to check first that

$$
\sigma_{1} z_{p}^{k}=\eta \tau z_{p}^{k} \in B_{p}(L)
$$

for every cycle $z_{p}^{k}$ of $K$. Now if $K$ is torsion-free in a particular dimension $p$, then $\tau z_{p}^{k} \in B_{p}(K)$, by property (iii) of $\tau$, hence a fortiori $\sigma_{1} z_{p}^{k}=\eta \tau z_{p}^{k} \in B_{p}(L)$. If $K$ is not torsion-free in dimension $p$, then $\beta \geqq p$ by the definition of $\beta$, and $p \geqq 1$, since a simplicial complex is torsion-free in dimension zero. Accordingly, $z_{p}^{k}$ lies in the subcomplex $S$, and bounds in the cone $\Gamma$ and hence also in $L$. Thus $\eta z_{p}^{k} \in B_{p}(L)$. On the other hand, since $K$ is not torsion-free in dimension $p$, we have $\tau z_{p}^{k}=z_{p}^{k}$ by property (iv) of $\tau$. It follows that $\sigma_{1} z_{p}^{k}=\eta \tau z_{p}^{k}=\eta z_{p}^{k}$ $\in B_{p}(L)$.

We shall show presently that the relation $\sigma_{1} \simeq \sigma_{2}$ fails to hold. Indeed, assume that there exists a homotopy operator

$$
D: C_{p}(K) \rightarrow C_{p+1}(L)
$$

for the pair $\sigma_{1}, \sigma_{2}$. Since $\sigma_{2}$ is the zero-homomorphism, we would then have identically

$$
\partial D c_{p}^{k}+D \partial \stackrel{k}{c_{p}}-\dot{\eta} \tau c_{p}^{k}=0
$$

for all chains $c_{p}^{k}$ of $K$. A contradiction is now readily obtained in the following manner. Since by assumption $K$ has torsion in dimension $\beta$, we have a particular cycle $z_{\beta}^{k}$ and a nonzero integer $n$, such that $n z_{\beta}^{k}$ bounds in $K$ :

$$
n z_{\beta}^{k}=\partial c_{\beta+1}^{k}, \quad n \neq 0,
$$

while on the other hand

$$
z_{\beta}^{k} \text { does not bound in } K \text {. }
$$


We have then by (4) the relation

$$
\partial D c_{\beta+1}^{k}+D \partial c_{\beta+1}^{k}-\eta \tau c_{\beta+1}^{k}=0 .
$$

Now $D c_{\beta+1}^{k}$ is a $(\beta+2)$-chain of $L$. Hence, by the remark (b) in $\S 5$, we can write

$$
D c_{\beta+1}^{k}=\eta d_{\beta+2}^{k} \text {. }
$$

(5), (7) yield (since $\eta$ commutes with $\partial$ ):

$$
\eta \partial d_{\beta+2}^{k}+n D z_{\beta}^{k}-\eta \tau c_{\beta+1}^{k}=0 .
$$

Denoting by $\pi: L \rightarrow K$ the projection from $L$ onto $K$, and applying $\pi$ on the left in (9), we get

$$
\partial d_{\beta+2}^{k}+n \pi D z_{\beta}^{k}-\tau c_{\beta+1}^{k}=0 .
$$

Now $\pi D z_{\beta}^{k}=e_{\beta+1}^{k}$, a $(\beta+1)$-chain in $K$. Making this substitution in $(10)$, and applying the boundary operator $\partial$, we obtain

$$
n \partial e_{\beta+1}^{k}-\partial \tau c_{\beta+1}^{k}=0 .
$$

Now $\partial \tau c_{\beta+1}^{k}=\tau \partial c_{\beta+1}^{k}=n \tau z_{\beta}^{k}$ by (5). Since $K$ does have torsion in dimension $\beta$, we have $\tau z_{\beta}^{k}=z_{\beta}^{k}$ by property (iv) of $\tau$. Thus (11) yields

$$
n \partial e_{\beta+1}^{k}-n z_{\beta}^{k}=0 .
$$

Since $n \neq 0$, it follows that $z_{\beta}^{k}=\partial e_{\beta+1}^{k}$. Thus $z_{\beta}^{k}$ would bound in $K$, in contradiction with (6).

\section{BiBLIOGRAPHY}

1. S. Lefschetz, Algebraic topology, Amer. Math. Soc. Colloquium Publications, vol. 27, 1942.

2. P. Alexandroff and H. Hopf, Topologie, Berlin, Springer, 1935.

The OHio State University 